\title{
Structures and optical properties of solid hydrogen at ultrahigh pressures
}

\author{
Hitose Nagara \\ Division of Materials Physics, Graduate School of Engineering Science, Osaka University \\ Toyonaka, Osaka 560-8531, Japan \\ E-mail: nagara@mp.es.osaka-u.ac.jp
}

\begin{abstract}
We have studied the electronic energy bands for the structures whose primitive cell contains up to four molecules, with full optimization of the structures, based on the GGA and LDA band calculations. Above $250 \mathrm{GPa}$, the eventual optimal structure obtained by the GGA or the LDA calculation is Cmca, which is a layered structure with the molecular bonds lying in planes, and has a metallic band structure with no band gaps. The metallic property of the band structure still remains unchanged even if the molecular bonds in the plane of the Cmca were inclined such that the atoms in the molecule escape from the plane. The electronic bands of the Cmca and those of some other candidate structures are discussed in the light of recent experimental result. Effects of the occupation of electronic states on the predicted optimal structures are also studied.
\end{abstract}

PACS: 62.50.+p, 61.50.Ah, 71.30.+h

\section{Introduction}

Recent experimental study of the compressed hydrogen revealed nonmetallic behavior at pressures higher than $\sim 300 \mathrm{GPa}[1,2]$. They report a possibility of the metallization by the closure of the direct electronic band gaps at even higher pressures. The result contradicts many theoretical studies based on the first principles band calculations which predict the closure of indirect band gaps at lower pressures in energetically favorable structures [3-5], and caused a question about an ability of the band calculations to predict the structures of compressed hydrogen.

In spite of the well-known shortcoming of the band calculation based on the local density approximation (LDA) or its modification with the gradient correction (GGA), that is, underestimation of the electronic band gaps, recent first principles band calculations have achieved much success in predicting structures and properties of solids at ambient as well as at high pressures. In the study of compressed hydrogen, whether this shortcoming might hamper the prediction of the structures and mislead us into false structures or not is still unclear because of lacking available experimental data of the structure above $100 \mathrm{GPa}$.

In order to check this point at some levels, we have carried out some preliminary calculations and re-examined the structures which have been predicted to be most probable at high pressures, paying attention to the occupation of the electronic bands by the electrons. We perform full optimization of the structures above $250 \mathrm{GPa}$ in the GGA and the LDA and study the changes of the electronic bands for structures which appear in the course of the optimization. We compare the results with new type of the calculation [6] which is expected to overcome the shortcoming and discuss the band structures in the light of very recent optical measurements [1].

\section{New restrictions imposed on the structures}

The results of the optical experiment [1] impose new restrictions on the structures from $150 \mathrm{GPa}$ to at least $320 \mathrm{GPa}$. The first important point to be mentioned is that the softening of the vibron frequency seems to occur continuously with increasing pressure. No jump of the frequency nor the change of the slope have been observed, which means no drastic changes of the structure. If the structure changes at all, it should be accompanied by a very small change of the vibron frequency. The second point is that the experiment reports the features characteristic of a direct band gap, which means the top of the valence band and the bottom of the conduction band are located at the same place in the Brillouin zone. 
In earlier experiments, the vibron frequencies and the optical properties at pressures over $250 \mathrm{GPa}$ were reported and the pressure of direct-gap closure has also been estimated from the optical data $[7,8]$. The recent optical measurements show the pressures and the character of the optical absorption more clearly. These new results impose important restrictions on the structures of solid hydrogen above the well-known $150 \mathrm{GPa}$ transition. Bearing these points in mind, we examine again some of the structures which have been theoretically studied so far.

\section{Calculations and results}

The structures which can be transformed continuously among them are $P c a 2_{1}, C m c 2_{1}, C m c a$, and Pbca family, which are shown in Fig. 1. Starting from one of those structures, the others are obtained by changing the molecular centers and the molecular orientation continuously.

At pressures lower than $200 \mathrm{GPa}$, the candidates of the most probable structure are $C m c 2_{1}, P c a 2_{1}$ with hcp molecular centers or its slight modifications [3,4]. The structure, however, becomes unstable at pressures higher than about $200 \mathrm{GPa}$ according to theoretical calculations [3-5]. In those calculations, they used the methods based on the LDA or the GGA which has a shortcoming of underestimating band gaps, resulting in the closure of indirect band gaps at around $200 \mathrm{GPa}$. Optimization of the structure is a delicate problem and the shortcoming might mislead us into the false optimal structures because of the false occupation of the states by the electrons whose energy is in
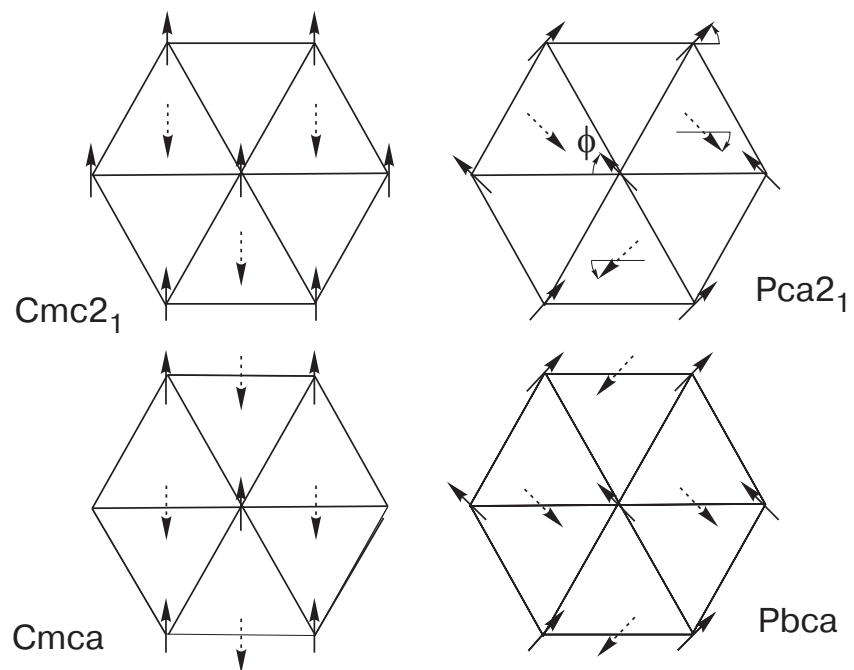

Fig. 1. The structures Pca2 $2_{1}, C m c 2_{1}, P b c a$, and Cmca. Arrows show the directions of molecular axes whose direction consigns with the $z$ axes are positive. These structure can be mutually transformed by continuously changing the molecular centers and orientations. the vicinity of the Fermi level. To investigate the effect of the electron occupation, we performed the calculations restricting the electron occupation.

We carried out band calculations using the plane-wave basis functions with energy cut-off of 40 hartree and a pseudo-potential for the ionic potential. Throughout our calculations, we used the packaged codes ABINIT [9] and PHI98PP [10].

\subsection{Preliminary calculations under restricted and unrestricted electronic occupation}

To study the effect of the false occupation on the optimal structures, we performed following preliminary calculations. First we show an example in which the restricted electron occupation and the unrestricted one give different conclusions in the comparison of total energies.

We compare the energies of the $C m c 2_{1}$ and the $P c a 2_{1}$ structures with molecular centers fixed at the hcp sites and with $c / a$ fixed at the ideal hcp value (see Fig. 1). We used the unit cell containing 4 molecules for both structures. In the first calculation, we performed the usual calculations in which the electrons occupy the states with energies lower than the Fermi energy. And in the second one, we restricted the occupation of the electronic states to the lowest 4 branches at each k-point, which simulates the insulator type of the electronic occupation of the bands. Up to $r_{s}=1.25$, which corresponds to a pressure around $430 \mathrm{GPa}$, the lowest 4 branches of the electronic energy states in the $C m c 2_{1}$ and the Pca2 ${ }_{1}$ seem to be well separated from the 5 th branch. Here the $r_{s}$ is the density parameter which is defined by the radius of the sphere, in units of Bohr radius, whose volume is equal to the volume per electron.

Comparing the energies of these two structure, we find that the $C m c 2_{1}$ is of lower energy than the Pca2 1 in the case of the first calculation. On the other hand, in the case of the second calculation, the Pca2 ${ }_{1}$ becomes lower.

\subsection{Full optimization of the structures}

Above example shows the possibility that the false occupation of the electronic states might affect the optimal structures at each pressure. The change of the occupation occurs when the indirect band gaps disappear. Starting from some arrangements of the atomic positions, we then performed full optimization of the structures.

As a starting arrangement, we take the $P c a 2{ }_{1}$ structure with orthorhombic unit cell containing 4 molecules. We have set the molecular centers at ideal hcp lattice sites and at several points between that of the ideal hcp site and the molecular center of the Pbca 
structure (see Fig. 1). The optimization has been done at constant volumes, at $r_{s}=1.40,1.35,1.30$, and 1.25, which correspond to the pressures around 200, 270, 330 , and $430 \mathrm{GPa}$, respectively. The pressures are estimated from the volume dependence of the total energy obtained in our calculations. The LDA calculations show smoother convergence than the GGA ones which contain the calculation of the density gradient. In the optimization of the structures, some runs did not converge to any meaningful structures. This is probably due to some problems in the optimization codes.

All structures converged and obtained as optimal ones in our runs are of $\mathrm{Cmca}$ for $r_{s} \leq 1.35$. We note here that the compression seems to be nearly isotropic above $200 \mathrm{GPa}\left(r_{s} \leq 1.40\right)$. Although the molecular centers move from the ideal hcp sites to that of the Cmca, the orthorhombic unit cell is compressed isotropically. At highest density $r_{s}=1.25$, the $c / a$ of the orthorhombic lattice decreased about $3 \%$ and the $b / a$ (distance between the layers) increased about $3 \%$, with molecular bonds tilted about $76^{\circ}$ from the $z$ axes and the bond length $1.41 a_{0}$. They did not show any molecular dissociation at all densities studied. These results are same as those obtained from the usual calculations with no restriction of the band occupation for both GGA and the LDA.

\section{Changes of the electronic band structure}

We study then the changes in the electronic band structures for those structures studied in the optimization process. The $C m c 2_{1}$ structure is also a layered structure with molecular bonds lying in the planes. The $C m c 2_{1}$ space group holds for any position of the molecular center between the hcp site and the molecular center of the Cmca. When the molecular bond is inclined in a certain way in the $C m c 2_{1}$ and two atoms of the molecule are off the plane, the structure becomes $P c a 2_{1}$. For the extreme case of the Pca2 $2_{1}$ in which the molecular center is moved to that of the Cmca, the space group becomes Pbca.

To compare the band structures we take the nonprimitive unit cell containing 4 molecules for all structures studied. All structures, except for the Cmca, have the band structure in which the lowest 4 branches are well separated from higher ones up to the highest densities $r_{s}=1.25$. The indirect band gaps, however, closes at much lower densities. The gaps becomes wider when the molecules in the $C m c 2_{1}$ are tilted toward the $P c a 2_{1}$ structure.

The $\mathrm{Cmca}$ has the band structure completely different from the others, where the band structure has no band gaps. The lowest 4 branch touches the higher ones at some points on the $\Sigma$-, $\Delta$ - and $c$-lines. The nature of the touching is line-type [3]. We show in
Fig. 2 the band structure of the Cmca taking the nonprimitive unit cell. Even when the molecular bonds are tilted towards the Pbca structure, the metallic band character still remains unchanged, though the changes of the distances between the branches are observed at several points in the Brillouin zone. In Fig. 2, we compare the band structure of the Cmca with that of the $P b c a$ at $r_{s}=1.25$.

It should be noted that the touching of the branches are due to the symmetry properties of the structure, independent of the approximation GGA or LDA used in the calculations. The distance among the branches depends on the approximation.

\section{Discussion and summary}

We discuss these results in the light of the recent optical measurements. Although the Cmca is energetically most favorable, it may be excluded because of the metallic character of band structure. The large fluctuation of the molecular orientation remaining in the Cmca structure [11] might change the electronic
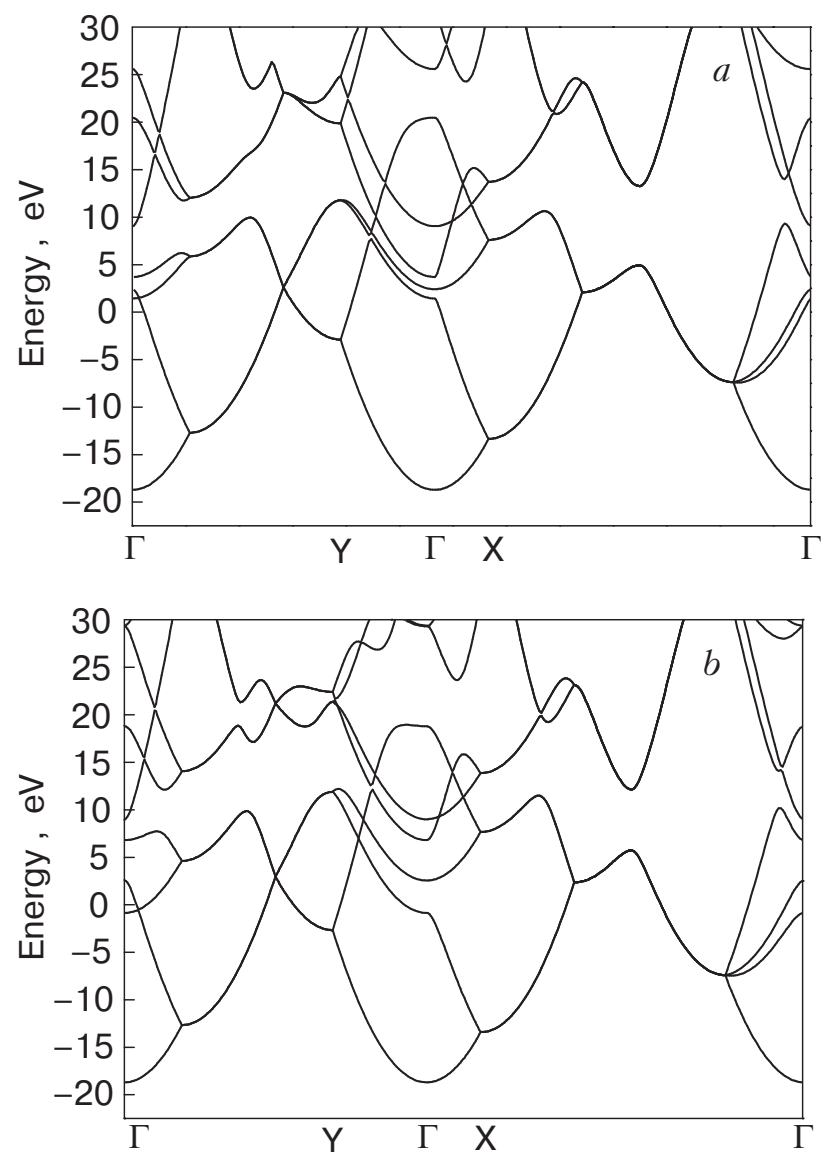

Fig. 2. The electronic band structures for the Cmca (a) and the Pbca (b) at $r_{s}=1.25$. To compare the changes in the energy bands, we used the nonprimitive unit cell containing 4 molecules for the Cmca structure whose primitive cell contains 2 molecules. 
bands. The metallic character of the electronic bands of the $P b c a$, however, may offer some negative insight into the changes of the band structure due to the fluctuation, although the movement from the Cmca to the $\mathrm{Pbca}$ is coherent. The possible fluctuation of the molecular center leaving the positions of the Cmca toward those of the $C m c 2_{1}$, however, might lift the metallic character of the electronic bands. If the energetically favorable Cmca structure is denied, there will remain two possibilities. One is that there are some other structures which we have overlooked in our study. An example is a structure of larger unit cell [4]. The second possibility is the case that all of the present GGA and LDA based calculations fail to predict energetically favorable structures of compressed hydrogen. In that case, the structure might remain those which is close to the $C m c 2_{1}$ or $P c a 2_{1}$ up to at least $320 \mathrm{GPa}$. The possibility of the second case has been reported recently in the new type of calculation [6] which is designed to overcome the shortcoming of the GGA and the LDA, though the optimization of the structures has not been carried out by that type of calculations owing mainly to machine resources.

We mention here the low lying librational and phonon modes observed in Raman experiments [12]. The $P c a 2_{1}$ has 9 optical phonons and 8 librational modes which are Raman active, and the $C m c 2_{1}$ has 3 optical phonons and 4 librational such modes, while the $C m c a$ has only 4 librational modes with no Raman active optical phonons.

Finally we mention that the metallization, which is predicted to occur at $\sim 450 \mathrm{GPa}$ by the extrapolation of the absorption edge to higher pressure, might happen at some lower pressures when the metallization is due to the closure of the indirect band gaps [8].

We have studied the optimal structures and the electronic bands in those energetically favorable struc- tures. The new results of the optical measurements brought about new problems into the theoretical studies of compressed hydrogen.

\section{Acknowledgments}

This work is supported by Grant-in-Aid for COE Research (10CE2004) of the Ministry of Education, Culture, Sports, Science and Technology of Japan.

1. P. Loubeyre, F. Occelli, and R. LeToullec, Nature 416, 613 (2002).

2. C. Narayana, H. Luo, J. Orloff, and A.L. Ruoff, Nature 393, 46 (1998)

3. K. Nagao, T. Takezawa, and H. Nagara, Phys. Rev. B59, 13741 (1999).

4. J. Kohanoff, S. Scandolo, S. Gironcoli, and E. Tosatti, Phys. Rev. Lett. 83, 4097 (1999).

5. K.A. Jonson and N.W. Ashkroft, Nature 403, 632 (2000)

6. M. Stadele and R.M. Martin, Phys. Rev. Lett. 84, 6070 (2000).

7. H.K. Mao and R.J. Hemly, Science 244, 1462 (1989).

8. M. Hanfland, R.J. Hemly, and H.K. Mao, Phys. Rev. B43, 8767 (1991).

9. The ABINIT code is a common project of the Université Catholique de Louvain, Corning Incorporated, and other contributors, URL http://www.abinit.org.

10. M. Fuchs and M. Scheffler, Comput. Phys. Commun. 119, 67 (1999), URL http:// www.fhi-berlin.mpg.de/th/fhi98vd/fhi98PP/index. html.

11. H. Kitamura, S. Tsuneyuki, T. Ogitsu, and T. Miyake, Nature 404, 259 (2000).

12. A.F. Goncharov, R.J. Hemly, H.K. Mao, and J. Shu, Phys. Rev. Lett. 80, 101 (1998). 